\title{
Semi-global stabilization of linear discrete-time systems subject to input saturation via linear feedback : an ARE-based approach
}

\section{Citation for published version (APA):}

Lin, Z., Saberi, A., \& Stoorvogel, A. A. (1994). Semi-global stabilization of linear discrete-time systems subject to input saturation via linear feedback : an ARE-based approach. (Memorandum COSOR; Vol. 9417). Technische Universiteit Eindhoven.

\section{Document status and date:}

Published: 01/01/1994

\section{Document Version:}

Publisher's PDF, also known as Version of Record (includes final page, issue and volume numbers)

\section{Please check the document version of this publication:}

- A submitted manuscript is the version of the article upon submission and before peer-review. There can be important differences between the submitted version and the official published version of record. People interested in the research are advised to contact the author for the final version of the publication, or visit the DOI to the publisher's website.

- The final author version and the galley proof are versions of the publication after peer review.

- The final published version features the final layout of the paper including the volume, issue and page numbers.

Link to publication

\footnotetext{
General rights

- You may freely distribute the URL identifying the publication in the public portal. follow below link for the End User Agreement:

www.tue.nl/taverne

\section{Take down policy}

If you believe that this document breaches copyright please contact us at:

openaccess@tue.nl

providing details and we will investigate your claim.
}

Copyright and moral rights for the publications made accessible in the public portal are retained by the authors and/or other copyright owners and it is a condition of accessing publications that users recognise and abide by the legal requirements associated with these rights.

- Users may download and print one copy of any publication from the public portal for the purpose of private study or research.

- You may not further distribute the material or use it for any profit-making activity or commercial gain

If the publication is distributed under the terms of Article $25 \mathrm{fa}$ of the Dutch Copyright Act, indicated by the "Taverne" license above, please 
EINDHOVEN UNIVERSITY OF TECHNOLOGY

Department of Mathematics and Computing Science

Memorandum COSOR 94-17

Semi-Global Stabilization of Linear

Discrete-Time Systems Subject to

Input Saturation via Linear Feedback

- An ARE-based Approach

Zongli Lin

A. Saberi

A.A. Stoorvogel

Eindhoven, June 1994

The Netherlands 


\title{
Semi-Global Stabilization of Linear Discrete-Time Systems Subject to Input Saturation via Linear Feedback
}

\section{- An ARE-Based Approach}

\section{Zongli Lin}

Dept. of Applied Mathematics and Statistics

SUNY at Stony Brook

Stony Brook, NY 11794-3600

U.S.A.

\author{
Ali Saberi \\ School of Electrical Engineering \\ and Computer Science \\ Washington State University \\ Pullman, WA 99164-2752
}

U.S.A.

\author{
Anton A. Stoorvogel \\ Department of Mathematics \\ and Computing Science \\ Eindhoven Univ. of Technology \\ P.O. Box 513, $5600 \mathrm{MB}$ Eindhoven \\ The Netherlands
}

\begin{abstract}
We revisit the problem of semi-global stabilization of linear discrete-time systems subject to input saturation and give a Riccati equation-based approach to the proof of a fact we established earlier ([3]), i.e., a linear discrete-time system subject to input saturation is semi-globally stabilizable via linear feedback as long as the linear system in the absence of the saturation is stabilizable and detectable and all its open loop poles are located inside or on the unit circle. Moreover, we drastically relax the requirements on the characteristic of the saturation elements as imposed in our earlier work [3].
\end{abstract}




\section{Introduction}

In this paper we revisit the problem of stabilizing a general linear system which is subject to input saturation. Over the last few years, there has been rather strong interest in this problem, possibly due to a wide recognition of the inherent constraints on the control input. As a result, several important results have appeared in the open literature. In the continuous-time setting, a recent result due to Sontag and Sussmann ([8]) shows that only linear stabilizable systems having no open-loop poles with positive real parts can be globally asymptotically stabilized by a bounded control. Another interesting aspect of this problem was shown by Fuller ([1]) and more recently by Sussmann and Yang ([10]). They showed that, for a system consisting of a chain of integrators of length $n$ where $n \geq 3$ which is subject to input saturation, there does not exist a linear control law that globally asymptotically stabilizes the given system. The implication of the results of [1], [8] and [10] is apparent and can be summarized as follows: Given a linear system which is subject to input saturation, the global asymptotic stabilization can be achieved if and only if all the poles of the given system are in the closed left half plane, and, even then, in general one must use nonlinear control and only very simple cases can be handled via linear feedback control laws. (An interesting nonlinear control law of the nested saturation type for global asymptotic stabilization of a chain of integrators subject to input saturation was proposed in [11] which was later extended to the general class of systems in [9].) In the discrete-time setting, an analogous result that a linear discrete-time system subject to input saturation can be globally asymptotically stabilized via feedback if and only if all its poles are located inside or on the unit circle has recently been established by Yang [13]. A nonlinear globally stabilizing control law for such a system is also explicitly constructed in [13]. More recently, we have learned that an analogy of the "negative result" for continuous time systems with saturating input ([1], [10]) has also been established in the discrete-time case ([14]). Namely, it is shown that similar to the continuous-time case, in general, for global asymptotic stabilization one must resort to nonlinear control laws.

Recently in [2] and [3], we have introduced the concept of semi-global analysis and design for linear systems subject to input saturation, in continuous-time as well as in discrete-time. The semi-global framework for analysis and design deals with techniques and methods of analysis and designs that are valid in an a priori given region of operation. This is in contrast with the traditional global viewpoint of analysis and design which requires methods and design techniques that are valid globally. The semi-global approach to the design of a system with saturating inputs is a very appealing concept since every plant model is valid only on a specific finite region of operation. We should emphasize that a key notion in semi- 
global design techniques is that the region of operation is a priori specified and this region can be chosen arbitrarily large.

A surprising result obtained in our work [2] and [3] is that linear feedback laws can be used for semi-global stabilization of linear continuous-time (discrete-time) systems subject to input saturation as long as the linear system in the absence of saturation is stabilizable and detectable and all its open loop poles are located in the closed left half plane (inside or on the unit circle). This is in contrast with global stabilization which cannot be achieved in general by linear feedback. In [2] and [3], we explicitly constructed such semi-globally stabilizing linear feedback laws with the use of a low-gain feedback design technique. These low-gain feedback laws were constructed in such a way that the control input does not saturate for any a priori given (arbitrarily large) bounded set of initial conditions. As a result, the closed-loop system operates as a linear system. The low-gain design technique introduced in [2] and [3] is based on eigenstructure assignment and is referred to as a direct design of low-gain controllers. More recently, for continuous-time case, methods utilizing $\mathrm{H}_{2}$ and $H_{\infty}$ optimal control theory for designing low-gain controllers have been proposed in [4] and [12] respectively. These methods are referred to as algebraic Riccati equation (ARE) based design of low-gain controllers. While the direct method is numerically superior, the ARE-based method is more compact and conceptually appealing. The goal of this paper is to provide an ARE-based design for low-gain controllers that semi-globally stabilize the same class of linear systems subject to input saturation as in [3] as well as relaxing the requirements on the saturation characteristic as imposed in [3]. We would like to mention that this work is not a simple translation of its continuous counterpart [12] into the discrete-time language. As a matter of fact, much effort has been made in relaxing the requirements on the saturation characteristic $\sigma$. In comparison with [12], we only require that in a neighborhood of the origin, each component of $\sigma$ lies inside any linear sector of first and third quadrant. Hence differentiability of $\sigma$ at the origin is not required. Moreover, only the knowledge of this linear sector is needed in the design.

The rest of this paper is organized as follows. In Section 2 we define the problems at hand. The solutions to these problems are the main results of this paper and are presented in Section 3. An example is given in Section 4 to demonstrate our state feedback results. We draw a brief concluding remark in the final Section 5 .

\section{Problem Statement}

We consider a class of nonlinear discrete-time systems commonly known as linear systems subject to input saturation. These systems are formed by cascading linear systems with 
memory-free input nonlinearities of saturation type,

$$
\begin{aligned}
x(k+1) & =A x(k)+B \sigma(u(k)) \\
y(k) & =C x(k)
\end{aligned}
$$

where $x \in \mathbf{R}^{n}$ is the state, $u \in \mathbf{R}^{m}$ is the control input to the saturater, $y \in \mathbf{R}^{p}$ is the measurement output, and $\sigma: \mathbf{R}^{m} \rightarrow \mathbf{R}^{m}$ is a saturation function defined as follows,

Definition 2.1. A function $\sigma: \mathbf{R}^{m} \rightarrow \mathbf{R}^{m}$ is called a saturation function if

1. $\sigma(u)$ is decentralized, i.e., $\sigma(u)=\left[\begin{array}{llll}\sigma_{1}\left(u_{1}\right) & \sigma_{2}\left(u_{2}\right) & \cdots & \sigma_{m}\left(u_{m}\right)\end{array}\right]^{\prime}$;

2. $s \sigma_{i}(s)>0$ whenever $s \neq 0$ and $\sigma(0)=0$;

3. There exist $\Delta>0$ and $b>b_{1}>0$ such that

$$
b_{1} \leq \frac{\sigma_{i}(s)}{s} \leq b, \quad 0<|s| \leq \Delta
$$

Remark 2.1. Graphically, the saturation function resides in the first and third quadrants and there exist $\Delta>0$ and $b>b_{1}>0$ such that for $|s| \leq \Delta$, the saturation function lies in the linear sector between the graphs graphs $\left(s, b_{1} s\right)$ and $(s, b s)$, which implies that,

$$
\begin{gathered}
s\left[\sigma_{i}(s)-b_{1} s\right] \geq 0 \\
\left|\sigma_{i}(s)-b_{1} s\right| \leq\left(b-b_{1}\right)|s|
\end{gathered}
$$

For notational simplicity, but without loss of generality, we will assume throughout this paper that $b_{1}=1$.

Remark 2.2. It follows directly from Definition 2.1 that the functions $\sigma(s)=s, \arctan (s)$, $\tanh (s)$ and the standard saturation function $\sigma(s)=\operatorname{sign}(s) \min \{|s|, 1\}$ are all saturation functions as defined in Definition 2.1. Moreover, functions like $\sigma(s)=2 s+s \sin (1 / s)$ with $\sigma(0)=0$, which are not even one-sided differentiable at the origin, also satisfy the conditions given in Definition 2.1 .

Definition 2.2. The set of all saturation functions that satisfy Definition 2.1 with a fixed triple of constants $\Delta, b_{1}=1$ and $b$ is denoted by $\mathcal{S}(\Delta, b)$.

We also make the following assumptions on the matrix triple $(A, B, C)$, Assumption 2.1. All the eigenvalues of $A$ are located inside or on the unit circle. 
Assumption 2.2. The pair $(A, B)$ is stabilizable;

Assumption 2.3. The pair $(A, C)$ is detectable.

We now define the problems to be solved in this paper.

Definition 2.3. For the system (2.1)-(2.2) with $\sigma \in \mathcal{S}(\Delta, b)$, where $\Delta$ and $b$ are some known positive constants, the semi-global exponential stabilization problem via linear state feedback is defined as follows. For any a priori given (arbitrarily large) bounded set $\mathcal{W} \subset \mathbf{R}^{n}$, find a state feedback law $u(k)=-F x(k)$ such that, for each $\sigma \in \mathcal{S}(\Delta, b)$, the equilibrium $x=0$ of the closed-loop system is locally exponentially stable and $\mathcal{W}$ is contained in its domain of attraction.

Definition 2.4. For the system (2.1)-(2.2) with $\sigma \in \mathcal{S}(\Delta, b)$, where $\Delta$ and $b$ are some known positive constants, the semi-global exponential stabilization problem via linear measurement feedback is defined as follows. For any a priori given (arbitrarily large) bounded set $\mathcal{W} \subset \mathbf{R}^{2 n}$, find a linear dynamic measurement feedback control law:

$$
\begin{aligned}
z(k+1) & =G z(k)+H y(k), \quad z \in \mathbf{R}^{n} \\
u(k) & =M z(k)+N y(k)
\end{aligned}
$$

such that for each $\sigma \in \mathcal{S}(\Delta, b)$, the equilibrium $(0,0)$ of the closed-loop system consisting of the system (2.1)-(2.2) and the controller (2.5)-(2.6) is locally exponentially stable and $\mathcal{W}$ is contained in its domain of attraction.

\section{Main Results}

Our main results on semi-global stabilization of the system (2.1)-(2.2) and their proof will be based on the following two lemmas regarding the properties of the algebraic Riccati equation (3.1).

Lemma 3.1. Let Assumptions 1 and 2 hold. Then, for any $\varepsilon>0$, there exists a unique matrix $P(\varepsilon)>0$ which solves the following algebraic Riccati equation:

$$
P=A^{\prime} P A+\varepsilon I-A^{\prime} P B\left(B^{\prime} P B+I\right)^{-1} B^{\prime} P A
$$

and is such that $A-B\left(B^{\prime} P(\varepsilon) B+I\right)^{-1} B^{\prime} P(\varepsilon) A$ is asymptotically stable. Moreover,

$$
\lim _{\varepsilon \rightarrow 0} P(\varepsilon)=0
$$


Proof. Existence and uniqueness of such a solution for $\varepsilon>0$ follows from [5]. For $\varepsilon=0$, it is trivial to see that (3.1) has a solution $P=0$ which is semi-stabilizing. Again from [5], this semi-stabilizing is unique. Finally, the fact that $\lim _{\varepsilon \rightarrow 0} P(\varepsilon)=0$ follows readily from the standard continuity arguments.

Lemma 3.2. Let Assumptions 1 and 2 hold. Then, there exists an $\varepsilon^{*}>0$ such that, for $\varepsilon \in\left(0, \varepsilon^{*}\right]$

$$
\left\|P^{\frac{1}{2}}(\varepsilon) A P^{-\frac{1}{2}}(\varepsilon)\right\| \leq \sqrt{2}
$$

Proof. Pre- and Post-multiplying both sides of the ARE (3.1) by $P^{-\frac{1}{2}}(\varepsilon)$, we obtain

$$
P^{-\frac{1}{2}}(\varepsilon) A^{\prime} P^{\frac{1}{2}}(\varepsilon)\left[I-P^{\frac{1}{2}}(\varepsilon) B\left(B^{\prime} P(\varepsilon) B+I\right)^{-1} B^{\prime} P^{\frac{1}{2}}(\varepsilon)\right] P^{\frac{1}{2}}(\varepsilon) A P^{-\frac{1}{2}}(\varepsilon)=I-\varepsilon P^{-\frac{1}{2}}(\varepsilon)
$$

By Lemma 3.1, $\lim _{\varepsilon \rightarrow 0} P(\varepsilon)=0$, hence it follows from (3.4) that there exists an $\varepsilon^{*}>0$ such that for all $\varepsilon \in\left(0, \varepsilon^{*}\right]$,

$$
P^{-\frac{1}{2}}(\varepsilon) A^{\prime} P(\varepsilon) A P^{-\frac{1}{2}}(\varepsilon) \leq 2 I-2 \varepsilon P^{-\frac{1}{2}}(\varepsilon) \leq 2 I
$$

which implies that

$$
\lambda_{\max }\left(P^{-\frac{1}{2}}(\varepsilon) A^{\prime} P(\varepsilon) A P^{-\frac{1}{2}}(\varepsilon)\right) \leq 2
$$

and completes the proof.

Our main results in this paper are now formulated in the following two theorems.

Theorem 3.1. Consider the system (2.1)-(2.2) with $\sigma \in \mathcal{S}(\Delta, b)$, where $\Delta$ and $b$ are arbitrary positive constants. If Assumptions 2.1 and 2.2 hold, then the semi-global exponential stabilization problem via linear state feedback, defined in Definition 2.3 , is solvable by the state feedback laws $u(k)=-\left(B^{\prime} P(\varepsilon) B+I\right)^{-1} B^{\prime} P(\varepsilon) A x(k)$, where $P(\varepsilon)>0$ is the unique solution of the algebraic Riccati equation (3.1). More specifically, for any a priori given (arbitrarily large) bounded set $\mathcal{W} \subset \mathbf{R}^{n}$, there exists an $\varepsilon^{*}>0$ such that, for each $\varepsilon \in\left(0, \varepsilon^{*}\right]$ and for each $\sigma \in \mathcal{S}(\Delta, b)$, the equilibrium $x=0$ of the closed loop system is locally exponentially stable and $\mathcal{W}$ is contained in its domain of attraction.

Proof. With the given state feedback laws, the closed-loop system takes the form of

$$
x(k+1)=A x(k)+B \sigma(u(k))=\left(A-B\left(B^{\prime} P B+I\right)^{-1} B^{\prime} P A\right) x(k)+B[\sigma(u(k))-u(k)]
$$

where, for notational brevity, we have dropped the dependency on $\varepsilon$ of the matrix $P(\varepsilon)$.

It follows from (3.1) that

$$
\left(A-B\left(B^{\prime} P B+I\right)^{-1} B^{\prime} P A\right)^{\prime} P\left(A-B\left(B^{\prime} P B+I\right)^{-1} B^{\prime} P A\right)-P=-\varepsilon I-Q_{0}
$$


where $Q_{0}:=A^{\prime} P B\left(B^{\prime} P B+I\right)^{-2} B^{\prime} P A \geq 0$.

We now pick a Lyapunov function

$$
V(x(k))=x^{\prime}(k) P x(k)
$$

and let $c$ be a strictly positive real number such that

$$
c \geq \sup _{x \in \mathcal{W}, \varepsilon \in(0,1]} x^{\prime} P x
$$

The right hand side is well defined since $\lim _{\varepsilon \rightarrow 0} P(\varepsilon)=0$ by Lemma 3.1 and $\mathcal{W}$ is bounded. Let $\varepsilon_{1}^{*}$ be such that for all $\varepsilon \in\left(0, \varepsilon_{1}^{*}\right], x \in L_{V}(c)$ implies that $\left\|\left(B^{\prime} P B+I\right)^{-1} B^{\prime} P A x\right\| \leq \Delta$, where the level set $L_{V}(c)$ is defined as $L_{V}(c)=\left\{x \in \mathbf{R}^{n}: V(x) \leq c\right\}$. Such an $\varepsilon_{1}^{*}$ exists because of Lemma 3.2 and the fact $\lim _{\varepsilon \rightarrow 0} P(\varepsilon)=0$.

The evaluation of the deference of $V$ along the trajectory of the closed-loop system (3.7), using (3.8) and Remark 2.1, shows that for all $x \in L_{V}(c)$,

$$
\begin{aligned}
V(x(k+1))- & V(x(k))=-x^{\prime}(k)\left(\varepsilon I+Q_{0}\right) x(k)+[\sigma(u(k))-u(k)]^{\prime} B^{\prime} P B[\sigma(u(k))-u(k)] \\
& +2 x^{\prime}(k)\left(A-B\left(B^{\prime} P B+I\right)^{-1} B^{\prime} P A\right)^{\prime} P B[\sigma(u(k))-u(k)] \\
\leq & -\varepsilon x^{\prime}(k) x(k)-u^{\prime}(k) u(k)+[\sigma(u(k))-u(k)]^{\prime} B^{\prime} P B[\sigma(u(k))-u(k)] \\
& +2 x^{\prime}(k) A^{\prime} P B\left(B^{\prime} P B+I\right)^{-1}[\sigma(u(k))-u(k)] \\
= & -\varepsilon x^{\prime}(k) x(k)-u^{\prime}(k) u(k)+[\sigma(u(k))-u(k)]^{\prime} B^{\prime} P B[\sigma(u(k))-u(k)] \\
& -2 u^{\prime}(k)[\sigma(u(k))-u(k)] \\
\leq & -\varepsilon x^{\prime}(k) x(k)-u^{\prime}(k) u(k)+(b-1)^{2} \lambda_{\max }\left(B^{\prime} P B\right) u^{\prime}(k) u(k)
\end{aligned}
$$

Again recalling that $\lim _{\varepsilon \rightarrow 0} P=0$, we easily see that there exists an $\varepsilon^{*} \in\left(0, \varepsilon_{1}^{*}\right]$ such that, for all $\varepsilon \in\left(0, \varepsilon^{*}\right],(b-1)^{2} \lambda_{\max }\left(B^{\prime} P B\right)-1 \leq 0$. This shows that for any $\varepsilon \in\left(0, \varepsilon^{*}\right]$,

$$
x \in L_{V}(c) \Longrightarrow V(x(k+1))-V(x(k)) \leq-\varepsilon x^{\prime}(k) x(k)
$$

which in turn shows that, for any $\varepsilon \in\left(0, \varepsilon^{*}\right]$, the equilibrium $x=0$ of the closed-loop system is locally exponentially stable and its domain of attraction contains the set $L_{V}(c)$. This completes our proof of Theorem 3.1 since $\mathcal{W} \subset L_{V}(c)$.

Theorem 3.2. Consider the system (2.1)-(2.2) with $\sigma \in \mathcal{S}(\Delta, b)$, where $\Delta$ and $b$ are arbitrary positive constants. If Assumptions 2.1, 2.2 and 2.3 hold, then the semi-global exponential stabilization problem via linear measurement feedback, defined in Definition 2.4, is solvable by the following family of linear measurement feedback laws

$$
\begin{aligned}
\hat{x}(k+1) & =\left(A-L C-B\left(B^{\prime} P(\varepsilon) B+I\right)^{-1} B^{\prime} P A\right) \hat{x}(k)+L y(k) \\
u(k) & =-\left(B^{\prime} P(\varepsilon) B+I\right)^{-1} B^{\prime} P A \hat{x}(k)
\end{aligned}
$$


where $P(\varepsilon)>0$ is the unique solution of the algebraic Riccati equation (3.1) and $L$ is any matrix such that $A-L C$ is asymptotically stable. More specifically, for any a priori given (arbitrarily large) bounded set $\mathcal{W} \subset \mathbf{R}^{2 n}$, there exists an $\varepsilon^{*}>0$ such that, for each $\varepsilon \in\left(0, \varepsilon^{*}\right]$ and for each $\sigma \in \mathcal{S}(\Delta, b)$, the equilibrium $(x, \hat{x})=(0,0)$ of the closed loop system is locally exponentially stable and $\mathcal{W}$ is contained in its domain of attraction.

Proof. With the given measurement feedback laws, the closed-loop system takes the following form

$$
\begin{aligned}
& x(k+1)=A x(k)+B \sigma\left(-\left(B^{\prime} P B+I\right)^{-1} B^{\prime} P A \hat{x}(k)\right) \\
& \hat{x}(k+1)=\left(A-L C-B\left(B^{\prime} P B+I\right)^{-1} B^{\prime} P A\right) \hat{x}(k)+L C x(k)
\end{aligned}
$$

where for the notational brevity, we have dropped the dependency on $\varepsilon$ of the matrix $P(\varepsilon)$. Letting $e=x-\hat{x}$, we rewrite the closed-loop system (3.14)-(3.15) as

$$
\begin{aligned}
& x(k+1)=\left(A-B\left(B^{\prime} P B+I\right)^{-1} B^{\prime} P A\right) x(k)-B u_{e}(k)+B[\sigma(u(k))-u(k)] \\
& e(k+1)=(A-L C) e(k)+B[\sigma(u(k))-u(k)]
\end{aligned}
$$

where we have defined $u_{e} \in \mathbf{R}^{m}$ as $u_{e}(k)=-\left(B^{\prime} P B+I\right)^{-1} B^{\prime} P A e(k)$. For later use, we also define $u_{x} \in \mathbf{R}^{m}$ as $u_{x}(k)=-\left(B^{\prime} P B+I\right)^{-1} B^{\prime} P A x(k)$. Clearly, $u(k)=u_{x}(k)-u_{e}(k)$.

Let $P_{e}>0$ be the unique solution to the Lyapunov equation

$$
P_{e}=(A-L C)^{\prime} P_{e}(A-L C)+I
$$

Such a unique solution exists since $A-L C$ is stable. We then choose a Lyapunov function

$$
V(x(k), e(k))=x^{\prime}(k) P x(k)+\sqrt{\lambda_{\max }(P)} e^{\prime}(k) P_{e} e(k)
$$

Let $c$ be a strictly positive real number such that

$$
c \geq \sup _{(x, \hat{x}) \in \mathcal{W}, \varepsilon \in(0,1]}\left(x^{\prime} P x+\sqrt{\lambda_{\max }(P)} e^{\prime} P_{e} e\right)
$$

The right hand side is well defined since $\lim _{\varepsilon \rightarrow 0} P(\varepsilon)=0$ by Lemma 3.1 and $\mathcal{W}$ is bounded. Let $\varepsilon_{1}^{*}$ be such that for all $\varepsilon \in\left(0, \varepsilon_{1}^{*}\right],(x, e) \in L_{V}(c)$ implies that $\|\left(B^{\prime} P B+I\right)^{-1} B^{\prime} P A(x-$ $e) \| \leq \Delta$, where the level set $L_{V}(c)$ is defined as $L_{V}(c)=\left\{(x, e) \in \mathbf{R}^{2 n}: V(x) \leq c\right\}$. Such an $\varepsilon_{1}^{*}$ exists because of Lemma 3.2 and the fact $\lim _{\varepsilon \rightarrow 0} P(\varepsilon)=0$.

The evaluation of the deference of $V$ along the trajectory of the closed-loop system (3.16)-(3.17), using (3.8), (3.18) and Remark 2.1, shows that for all $(x, e) \in L_{V}(c)$, 


$$
\begin{aligned}
& V(x(k+1), e(k+1))-V(x(k), e(k)) \\
& =-u_{x}^{\prime}(k) u_{x}(k)+[\sigma(u(k))-u(k)]^{\prime} B^{\prime} P B[\sigma(u(k))-u(k)] \\
& -\varepsilon x^{\prime}(k) x(k)+2 x^{\prime}(k)\left(A-B\left(B^{\prime} P B+I\right)^{-1} B^{\prime} P A\right)^{\prime} P B[\sigma(u(k))-u(k)] \\
& -2 x^{\prime}(k)\left(A-B\left(B^{\prime} P B+I\right)^{-1} B^{\prime} P A\right)^{\prime} P B u_{e} u_{e}(k) \\
& +u_{e}^{\prime}(k) B^{\prime} B u_{e}(k)-2 u_{e}^{\prime}(k) B^{\prime} B[\sigma(u(k))-u(k)] \\
& -\sqrt{\lambda_{\max }(P)} e^{\prime}(k) e(k)+2 \sqrt{\lambda_{\max }(P)} e^{\prime}(k)(A-L C)^{\prime} P_{e} B[\sigma(u(k))-u(k)] \\
& +\sqrt{\lambda_{\max }(P)}[\sigma(u(k))-u(k)]^{\prime} B^{\prime} P_{e} B[\sigma(u(k))-u(k)] \\
& \leq-u_{x}^{\prime}(k) u_{x}(k)+(b-1)^{2} \lambda_{\max }\left(B^{\prime} P B\right) u^{\prime}(k) u(k)-2 u_{x}^{\prime}(k)[\sigma(u(k))-u(k)] \\
& +u_{e}^{\prime}(k) B^{\prime} B u_{e}(k)-2 u_{e}^{\prime}(k) B^{\prime} B[\sigma(u(k))-u(k)]-\sqrt{\lambda_{\max }(P)} e^{\prime}(k) e(k) \\
& -\varepsilon x^{\prime}(k) x(k)+2 \sqrt{\lambda_{\max }(P)} e^{\prime}(k)(A-L C)^{\prime} P_{e} B[\sigma(u(k))-u(k)] \\
& +2 u_{x}^{\prime}(k) u_{e}(k)+\sqrt{\lambda_{\max }(P)}[\sigma(u(k))-u(k)]^{\prime} B^{\prime} P_{e} B[\sigma(u(k))-u(k)] \\
& \leq-\varepsilon x^{\prime}(k) x(k)-u_{x}^{\prime}(k) u_{x}(k)+2(b-1)^{2} \lambda_{\max }\left(B^{\prime} P B\right)\left[u_{x}^{\prime}(k) u_{x}(k)+u_{e}^{\prime}(k) u_{e}(k)\right] \\
& +2(b-1)\left(1+\lambda_{\max }\left(B^{\prime} B\right)\right) u_{e}(k)^{\prime} u_{e}(k)+4(b-1)^{2}\left(1+\lambda_{\max }\left(B^{\prime} B\right)\right)^{2} u_{e}^{\prime}(k) u_{e}(k) \\
& +\frac{1}{4} u_{x}^{\prime}(k) u_{x}(k)+4 u_{e}^{\prime}(k) u_{e}(k)+\lambda_{\max }\left(B^{\prime} B\right) u_{e}(k)^{\prime} u_{e}(k)-\sqrt{\lambda_{\max }(P)} e^{\prime}(k) e(k) \\
& +\frac{1}{4} u_{x}^{\prime}(k) u_{x}(k)+2 \alpha \lambda_{\max }^{\frac{3}{2}}(P)\|A\|\|B\| e^{\prime}(k) e(k)+4 \alpha^{2} \lambda_{\max }(P) e^{\prime}(k) e(k) \\
& +\frac{1}{4} u_{x}^{\prime}(k) u_{x}(k)+2(b-1)^{2} \sqrt{\lambda_{\max }(P)} \lambda_{\max }\left(B^{\prime} P_{e} B\right)\left[u_{x}^{\prime}(k) u_{x}(k)+u_{e}^{\prime}(k) u_{e}(k)\right] \\
& =-\varepsilon x^{\prime}(k) x(k)-\sqrt{\lambda_{\max }(P)}\left(1-4 \alpha^{2} \sqrt{\lambda_{\min }(P)}-2 \alpha \lambda_{\max }(P)\|B\|\|A\|\right) e^{\prime}(k) e(k) \\
& -\left[\frac{1}{4}-2(b-1)^{2} \lambda_{\max }\left(B^{\prime} P B\right)-2(b-1)^{2} \sqrt{\lambda_{\max }(P)} \lambda_{\max }\left(B^{\prime} P_{e} B\right)\right] u_{x}^{\prime}(k) u_{x}(k) \\
& +\left[2(b-1)^{2} \lambda\left(B^{\prime} P B\right)+4(b-1)^{2}\left(1+\lambda_{\max }\left(B B^{\prime}\right)\right)^{2}+2(b-1)\left(1+\lambda_{\max }\left(B^{\prime} B\right)\right)\right. \\
& \left.+4+\lambda_{\max }\left(B^{\prime} B\right)+2(b-1)^{2} \sqrt{\lambda_{\max }(P)} \lambda_{\max }\left(B^{\prime} P_{e} B\right)\right] u_{e}^{\prime}(k) u_{e}(k)
\end{aligned}
$$

where we have denoted $\alpha:=(b-1)\left\|(A-L C)^{\prime} P_{e} B\right\|$.

Recalling that $\lim _{\varepsilon \rightarrow 0} P=0$ and $\alpha$ is independent of $\varepsilon$, we easily verify that there exists an $\varepsilon^{*} \in\left(0, \varepsilon_{1}^{*}\right]$ such that for all $\varepsilon \in\left(0, \varepsilon^{*}\right]$, the following hold,

$$
\begin{gathered}
\frac{1}{4}-2(b-1)^{2} \lambda_{\max }\left(B^{\prime} P B\right)-2(b-1)^{2} \sqrt{\lambda_{\max }(P)} \lambda_{\max }\left(B^{\prime} P_{\mathrm{e}} B\right) \geq 0 \\
4 \alpha^{2} \sqrt{\lambda_{\min }(P)}+2 \alpha \lambda_{\max }(P)\|B\|\|A\| \leq \frac{1}{4}
\end{gathered}
$$

and

$$
\left[2(b-1)^{2} \lambda\left(B^{\prime} P B\right)+4(b-1)^{2}\left(1+\lambda_{\max }\left(B B^{\prime}\right)\right)^{2}+2(b-1)\left(1+\lambda_{\max }\left(B^{\prime} B\right)\right)+4\right.
$$




$$
\left.+\lambda_{\max }\left(B^{\prime} B\right)+2(b-1)^{2} \sqrt{\lambda_{\max }(P)} \lambda_{\max }\left(B^{\prime} P_{e} B\right)\right] u_{e}^{\prime}(k) u_{e}(k) \leq \frac{\sqrt{\lambda_{\max }(P)}}{4} e^{\prime}(k) e(k)
$$

Hence, it follows from (3.21) that for all $\varepsilon \in\left(0, \varepsilon^{*}\right]$,

$(x, e) \in L_{V}(c) \Longrightarrow V(x(k+1), e(k+1))-V(x(k), e(k)) \leq-\varepsilon x^{\prime}(k) x(k)-\frac{\sqrt{\lambda_{\max }(P)}}{2} e^{\prime}(k) e(k)$ which shows that, for any $\varepsilon \in\left(0, \varepsilon^{*}\right]$, the equilibrium $(x, e)=(0,0)$ of the closed-loop system is locally exponentially stable and its domain of attraction contains the set $L_{V}(c)$. This completes our proof of Theorem 3.2 since $(x, \hat{x}) \in \mathcal{W}$ implies that $(x, e) \in L_{V}(c)$.

\section{An Example}

In this section we want to present a simple example to illustrate some of the results in this paper. Consider the following system:

$$
x(k+1)=\left(\begin{array}{cccc}
0 & 1 & 0 & 0 \\
0 & 0 & 1 & 0 \\
0 & 0 & 0 & 1 \\
-1 & 2 \sqrt{2} & -4 & 2 \sqrt{2}
\end{array}\right) x(k)+\left(\begin{array}{c}
0 \\
0 \\
0 \\
1
\end{array}\right) \sigma(u(k))
$$

which has repeated open loop poles at $\left\{\frac{\sqrt{2}}{2}+j \frac{\sqrt{2}}{2}, \frac{\sqrt{2}}{2}+j \frac{\sqrt{2}}{2}, \frac{\sqrt{2}}{2}-j \frac{\sqrt{2}}{2}, \frac{\sqrt{2}}{2}-j \frac{\sqrt{2}}{2}\right\}$. The only knowledge we have about $\sigma$ is that $\sigma \in \mathcal{S}(1,5)$. By Theorem 3.1, the problem of semi-global stabilization via linear state feedback is solvable. For example, for the a priori given set

$$
\mathcal{W}=\left\{x \in \mathbf{R}^{4}|| x_{i} \mid \leq 4\right\}
$$

we first choose a $c$ that satisfies (3.10). Since $P$ is increasing in $\varepsilon$ it is sufficient to choose:

$$
c=\sup _{x \in \mathcal{W}} x^{\prime} P(1) x \approx 1875.46
$$

We next pick $\varepsilon_{1}^{*}$ as

$$
\varepsilon_{1}^{*}=\sup \left\{\varepsilon \in(0,1]\|\|\left(B^{\prime} P B+I\right)^{-1} B^{\prime} P A x \| \leq 1 \quad \forall x \in L_{V}(c)\right\} \approx 9.33 \cdot 10^{-9}
$$

Finally we pick $\varepsilon^{*}$ to be

$$
\varepsilon^{*}=\sup \left\{\varepsilon \in\left(0, \varepsilon_{1}^{*}\right] \mid 16 \lambda_{\max }\left(B^{\prime} P B\right) \leq 1\right\}=\varepsilon_{1}^{*} \approx 9.33 \cdot 10^{-9}
$$

It then follows from the proof of Theorem 3.1 that, for each $\varepsilon \in\left(0, \varepsilon^{*}\right]$, the equilibrium $x=0$ of the closed-loop system under the linear state feedback $u=-\left(B^{\prime} P B+I\right)^{-1} B^{\prime} P A x$ is locally exponentially stable with $\mathcal{W}$ contained in its domain of attraction. For $\varepsilon=5 \cdot 10^{-9}$, we have

$$
u=\left(\begin{array}{llll}
0.0235 & -0.0501 & 0.0474 & -0.0168
\end{array}\right) x
$$




\section{Conclusions}

In this paper we have studied the problem of stabilizing discrete-time linear systems subject to input saturation. We have shown that low-gain linear feedback controllers can achieve semi-global stabilization. Moreover, the resulting controller can be obtained using very little information of the precise structure of the saturation. The latter is very important since in practice we often only know a region where the actuator will behave approximately linear. Our controller is very robust with respect to changes in the saturation function.

\section{References}

[1] A.T. Fuller, In the large stability of relay and saturated control systems with linear controllers, International Journal of Control, vol, 15, pp. 486-505, 1977.

[2] Z. Lin and A. Saberi, Semi-global exponential stabilization of linear systems subject to "input saturation" via linear feedbacks, Systems $\&$ Control Letters, vol. 21, pp.225239, 1993.

[3] Z. Lin and A. Saberi, Semi-global exponential stabilization of linear discrete-time systems subject to "input saturation" via linear feedbacks, to appear in Systems $\mathcal{G}$ Control Letters.

[4] Z. Lin, A.A. Stoorvogel and A. Saberi, "Output regulation for linear systems subject to input saturation," submitted for publication, 1993.

[5] A. Saberi, P. Sannuti and B.M. Chen, $\mathrm{H}_{2}$ Optimal Control, Prentice-Hall International, to appear.

[6] W.E. Schmitendorf and B.R. Barmish, Null controllability of linear systems with constrained controls, SIAM J. Control and Optimization, vol. 18, pp. 327-345, 1980.

[7] E.D. Sontag, An algebraic approach to bounded controllability of linear systems, International Journal of Control, vol. 39, pp. 181-188, 1984.

[8] E.D. Sontag ans H.J. Sussmann, Nonlinear output feedback design for linear systems with saturating controls, Proc. 29th IEEE Conf. Decision and Control, pp. 3414$3416,1990$.

[9] E.D. Sontag and Y. Yang, Global stabilization of linear systems with bounded feedback, Report SYCON-91-09, Rutgers Center for Systems and Control, 1991.

[10] H.J. Sussmann and Y. Yang, On the stabilizability of multiple integrators by means of bounded feedback controls, Report SYCON-91-01, Rutgers Center for Systems and Control, 1991. 
[11] A.R. Teel, "Global stabilization and restricted tracking for multiple integrators with bounded controls," System \& Control Letters, vol. 18, pp. 165-171, 1992.

[12] A.R. Teel, "Semi-global stabilization of linear controllable systems with input nonlinearities," to appear in IEEE Transaction on Automatic Control, 1993.

[13] Y. Yang, Global Stabilization of Linear Systems with Bounded Feedback, Ph.D dissertation, New Brunswick Rutgers, the State University of New Jersey, 1993.

[14] Y. Yang, Private Communications, 1993. 